\title{
Automated laser fluorescence analysis of randomly amplified polymorphic DNA: a rapid method for investigating nosocomial transmission of Acinetobacter baumannii
}

\author{
H. GRUNDMANN, CH. SCHNEIDER, H. V. TICHY*, R. SIMON*, I. KLARE $\dagger$, DORIS HARTUNG \\ and F. D. DASCHNER
}

Institut für Umweltmedizin und Krankenhaushygiene, Universitätsklinik Freiburg, Hugstetter Str. 55, 79106 Freiburg, - Fachgruppe Biologische Sicherheit, TÜV Energie und Umwelt GmbH, Robert-Bunsen-Str. 1, 79108 Freiburg, $\dagger$ Robert Koch Institut, Burgstr. 37, 38855 Wernigerode, Germany

\begin{abstract}
Summary. A rapid method for genotyping Acinetobacter baumannii based on PCRfingerprinting with fluorescent primers was evaluated. Automated laser fluorescence analysis (ALFA) enabled on-line generation of high resolution DNA-fingerprints during polyacrylamide gel electrophoresis of randomly amplified polymorphic DNA (RAPD) products. The results were in concordance with macro-restriction fragment patterns produced by pulsed-field gel electrophoresis (PFGE) of ApaI digests of chromosomal DNA. RAPDALFA was able to identify homologous strains suggestive of horizontal transmission in $<8 \mathrm{~h}$ after colonies were obtained on solid media, whereas PFGE analysis took $c$. $90 \mathrm{~h}$. Speed and digitised data format renders RAPD-ALFA attractive for routine in-house epidemiological screening of isolates from intensive care and other hospital units.
\end{abstract}

\section{Introduction}

Polymerase chain reaction (PCR)-based DNA fingerprinting is a well described method for taxonomic and epidemiological investigations of bacterial pathogens. ${ }^{1}$ In contrast to conventional target-specific PCR, arbitrarily primed PCR (AP-PCR) ${ }^{2}$ and randomly amplified polymorphic DNA (RAPD) ${ }^{3}$ analysis generally use a single, short arbitrarily chosen primer which anneals to multiple sites on the bacterial chromosome. Where these primers anneal sufficiently close together and in the correct orientation, intervening sequences are amplified in a PCR to give multiple products. Electrophoretic separation of these products yields patterns, or fingerprints, that may allow discrimination between strains at the subspecies level. Macrorestriction fragment analysis by pulsedfield gel electrophoresis (PFGE) has been reported to yield a very high discrimination of bacterial strains ${ }^{4-6}$ but is time consuming and, therefore, mainly used by large reference centres for outbreak investigations. RAPD provides a rapid and simple method suitable for in-house epidemiological screening of clinical isolates, especially those from critically ill or severely immunocompromised patients who are particularly vulnerable to nosocomial colonisation and infection. RAPD has been applied successfully to epidemiological investigations of a wide range of bacteria including Pseudomonas aeruginosa, Staphylococcus aureus, Clostridium difficile, Legionella spp., Klebsiella spp., Serratia marcescens and Acinetobacter baumannii. ${ }^{1,4}$ However, the application of RAPD to routine screening has been hampered by difficulties that arise when aligning the banding patterns of a large number of isolates obtained from different gels over long periods of time. Lack of reproducibility has also been reported to be a problem with RAPD and standardisation of reagents and equipment is vital. ${ }^{7,8}$

A novel method of data analysis for DNA fingerprinting based on RAPD combined with automated laser fluorescence analysis (ALFA) which includes pattern recognition by computed cluster analysis was evaluated. $A$. baumannii was chosen as a representative organism of epidemiological importance in hospitals. Results were compared with macro-restriction analysis of ApaI-digested chromosomal DNA by PFGE. 


\section{Materials and methods}

\section{Bacterial strains}

Fifteen isolates were included in this study. Four isolates (nos. 1-4) were associated with an outbreak on an intensive care unit at the Charite Hospital, Berlin. At the University Hospital, Freiburg, prospective isolates of $A$. baumannii were selected if they were both clearly associated with infection at the site of isolation and obtained from two or more patients during a common stay on the same ward, suggestive of nosocomial transmission. Between December 1993 and September 1994, 10 of a total of 39 A. baumannii isolates obtained by routine diagnostic investigations at the Departments of Internal Medicine and Paediatrics satisfied these criteria (nos. 5 and 7-15). These represented isolates from two patients with blood stream infections, six lower respiratory tract infections and two soft tissue infections. One isolate (no. 6) was from the hands of a nurse who cared for two neonates with pneumonia from whom $A$. baumannii was isolated from tracheal secretions (nos. 5 and 7). Identification of $A$. baumannii was based on standard biochemical reactions and the criteria of Bouvet and Grimont. ${ }^{9}$

\section{$R A P D-A L F A$}

Isolates were cultured overnight on blood agar (BBL, Heidelberg, Germany) containing horse blood $5 \%$. RAPD was performed in duplicate; two colonies of each isolate were analysed separately. Crude DNA extracts were prepared by a quick boil-lysis procedure. A single colony was resuspended in $100 \mu \mathrm{l}$ of $\mathrm{H}_{2} \mathrm{O}$ and heated to $95^{\circ} \mathrm{C}$ for $10-15 \mathrm{~min}$. Cell debris was sedimented by centrifugation and $2.5 \mu \mathrm{l}$ of the supernate provided template DNA for the amplification reaction. A single primer with the sequence $5^{\prime}-\mathrm{GAG}$ GGT GGC GGT TCT-3' (from the core region of bacteriophage M13) labelled with fluorescein at the $5^{\prime}$ end, was used. A total volume of $50 \mu 1$ of reaction mix contained $1 \times$ PCR buffer (Pharmacia, Freiburg, Germany), $1 \mu \mathrm{M}$ primer, $200 \mu \mathrm{M}$ of each dNTP, $3 \mathrm{mM}$ magnesium acetate, $0.5 \mathrm{IU}$ Taq-polymerase (Pharmacia) and target DNA. Amplification conditions consisted of 35 cycles of $94^{\circ} \mathrm{C}$ for $20 \mathrm{~s}, 50^{\circ} \mathrm{C}$ for $60 \mathrm{~s}$ and $72^{\circ} \mathrm{C}$ for $20 \mathrm{~s}$. The size marker was the $100-\mathrm{bp}$ ladder (Boehringer, Mannheim, Germany) previously labelled with fluorescent dUTP by the Klenow fill-in reaction. A fluorescence-labelled 100-bp fragment of lambda DNA (Pharmacia) and a 1064-bp PCR product from Escherichia coli small subunit rDNA, generated with the two consensus primers described by Barry and co-workers, ${ }^{10}$ were added to each sample as internal size standards.

Amplification products were analysed by a highspeed automated DNA fragment analysis device based upon the automated fluorescent DNA sequencer (A.L.F., Pharmacia), described by Ansorge et al. ${ }^{11}$
Briefly, fluorescently labelled amplification products pass a fixed laser beam during electrophoresis. Emitted light is detected by photodetectors and signals, collected every $2.5 \mathrm{~s}$, are digitised and sent to the computer for processing and storage. During electrophoresis data can be displayed as a fluorogram in real time on the computer screen.

Denaturing separating gels were prepared containing Hydrolink Long Ranger (A and T Biochemicals, Malvern, USA) $5 \%, 7 \mathrm{M}$ urea, (A.L.F. grade, Pharmacia) and $0.6 \times$ TBE $(1 \times$ TBE is $0.089 \mathrm{M}$ Tris, $0.089 \mathrm{M}$ boric acid and $0.002 \mathrm{M}$ EDTA). One $\mu$ l of amplification products was denatured in $5 \mu \mathrm{l}$ of stop solution (stop solution is dextran blue $5 \mathrm{mg} / \mathrm{ml}$ formamide) at $95^{\circ} \mathrm{C}$ for $5 \mathrm{~min}$ and then applied to the gel. The electrophoresis buffer was $0.6 \times \mathrm{TBE}$ and the temperature was kept constant at $45^{\circ} \mathrm{C}$. Stored fluorograms were normalised with the internal standards (100-bp, 1064-bp) and size markers as references by Fragment Manager Software (Pharmacia). Dendrograms for cluster analysis were based on similarity matrices calculated from the Pearson product-moment correlation coefficient computed by GelCompar software (Applied Maths, Kortrijk, Belgium).

\section{PFGE analysis}

For macro-restriction analysis, A. baumannii strains were grown overnight in Tryptic Soy Broth (Oxoid) to a density of $10^{9} \mathrm{cfu} / \mathrm{ml}$. Cells were sedimented by centrifugation, washed in $5 \mathrm{ml}$ of $10 \mathrm{~mm}$ Tris, $1 \mathrm{M}$ $\mathrm{NaCl}, \mathrm{pH} 7 \cdot 5$, and resuspended in $900 \mu \mathrm{l}$ of the same buffer. Five hundred $\mu \mathrm{l}$ of this suspension were mixed with an equal volume of low-melting agarose $1.3 \%$ and solidified in moulds. Agarose inserts were then transferred to lysis buffer containing proteinase K $0.05 \%$, sodium-laurylsarcosine $1 \%, 0.5 \mathrm{M}$ EDTA, pH 9.6, and cells were lysed at $56^{\circ} \mathrm{C}$ for $c .16 \mathrm{~h}$. The inserts were subsequently equilibrated in $10 \mathrm{~mm}$ Tris, $1 \mathrm{~mm}$ EDTA, pH 8.0 and stored at $4^{\circ} \mathrm{C}$ until use. All agarose inserts were then equilibrated with restriction buffer (Pharmacia) and digested by the rare-cutting endonuclease ApaI (Pharmacia) during overnight incubation at $37^{\circ} \mathrm{C}$. Slices of $c .1 \mathrm{~mm}$ from previously digested agarose plugs were inserted into gel pockets and sealed with low melting agarose. Electrophoresis was in agarose gels (Seakem, FMC BioProducts, Rockland, USA) $1 \% \mathrm{w} / \mathrm{v}$ in $0.5 \times \mathrm{TBE}$ with a $\mathrm{CHEF}$ DRII drive module (BioRad, Richmond, USA). Electrophoresis conditions consisted of two ramps in sequence: $\operatorname{ramp} \mathbf{A}$, with an initial switch time of $0.5 \mathrm{~s}$ and final switch time of $25 \mathrm{~s}$, was run for $20 \mathrm{~h}$, followed by ramp B, with an initial switch time of $30 \mathrm{~s}$ and final switch time of $60 \mathrm{~s}$, for $4 \mathrm{~h}$. The voltage was $200 \mathrm{~V}$ for both ramp types, and the temperature was kept constant at $14^{\circ} \mathrm{C}$. Fragments were stained with ethidium bromide and photographed with ultraviolet transillumination. 


\section{Results}

RAPD profiles of the 15 isolates of $A$. baumannii were displayed as fluorescence densitograms (fluoro- grams) by the automated DNA sequencer. Two colonies of each isolate were processed separately and electrophoresed in adjacent lanes. These duplicates revealed a similarity of $>91 \%$, when amplification

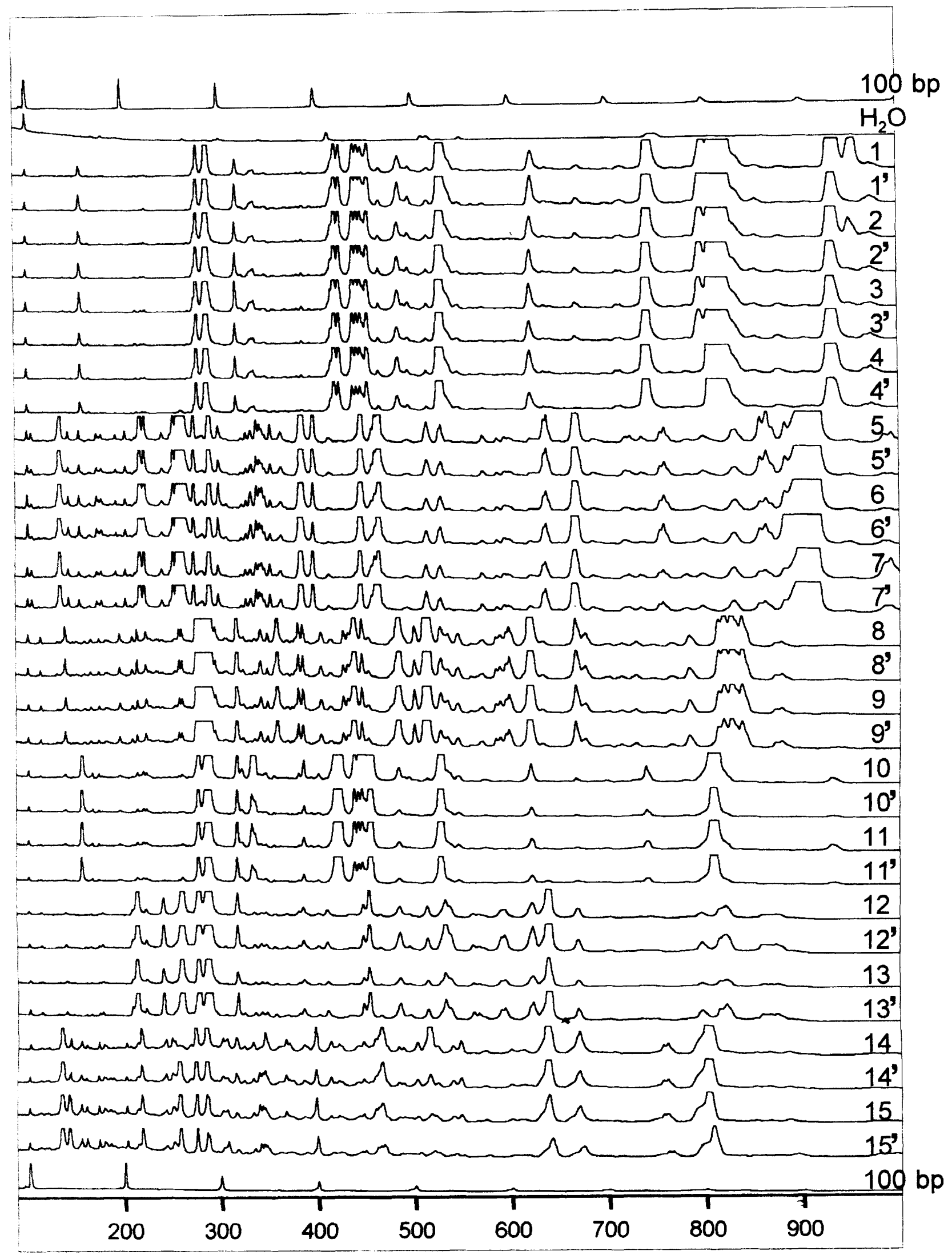

Fig. 1. Fluorescence densitogram of $A$. baumannii RAPD generated by the DNA sequencer. Strains: $1-4$, represent outbreak isolates from different patients ; $5-7$, patient isolates and a hand isolate from a nurse; $8-15$, were obtained from pairs of patients during a common stay on the same ward; ' indicates duplicates. 


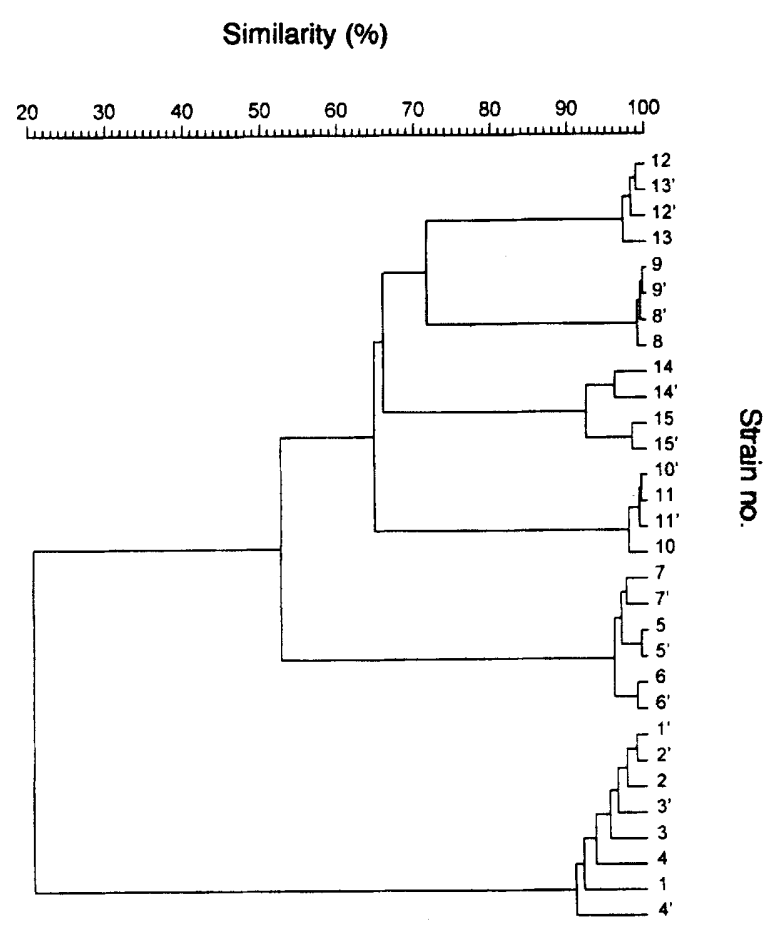

Fig. 2. Dendrogram of similarity values for strain specific amplification patterns based on fluorescence densities as depicted in fig. 1 .

profiles up to $1000 \mathrm{bp}$ were compared. Minor differences in fingerprinting patterns were caused by DNA fragments $>700$ bp which occasionally showed variable intensities as indicated in lanes 1 and $1^{\prime}, 2$ and $2^{\prime}$, and $5-7^{\prime}$ of the fluorogram (fig. 1). However, overall reproducibility remained $>90 \%$ after retesting of the same isolate 10 or 20 times (data not shown). If similarity analysis was confined to fragments $<700 \mathrm{bp}$, pattern similarity for duplicates was well above $95 \%$, indicating a high degree of reproducibility of the randomly primed amplification of the smaller products.

Automated similarity analysis of RAPD fluorescence profiles up to $1000 \mathrm{bp}$ from strains $1-4$ revealed a similarity of $92 \%$. These strains represented isolates from a single outbreak. Similarly, strains 5 and 7 , obtained from two patients, and strain 6 , from the hand culture of a nurse who had cared for both of these patients, had very similar amplification patterns (96\% similarity). The fluorescence profiles of the eight clinical isolates (strains 8-15) obtained from four pairs of patients infected with $A$. baumannii during common stays on the same ward revealed four pattern types in which the isolates clustered pairwise with similarity values of $98 \%, 98 \%, 96 \%$ and $93 \%$, respectively. Similarity values of $<72 \%$ discriminated between clusters of epidemiologically unrelated strains (fig. 2). The results generated by RAPD-ALFA agreed with those from macro-restriction analysis of chromosomal DNA after digestion with ApaI (fig. 3).

\section{Discussion}

A. baumannii is a frequent nosocomial pathogen often expressing a high degree of antimicrobial resistance. ${ }^{12,13}$ It is a member of the transient skin flora, a frequent contaminant of fomites and often isolated from water supplies. ${ }^{14}$ Both its ubiquitous occurrence and importance for hospital epidemiology warrant a screening technique that is able to identify epidemic strains at an early stage of dissemination. ${ }^{13}$ Therefore, A. baumannii represents a relevant candidate for the assessment of the efficiency of any method of strain

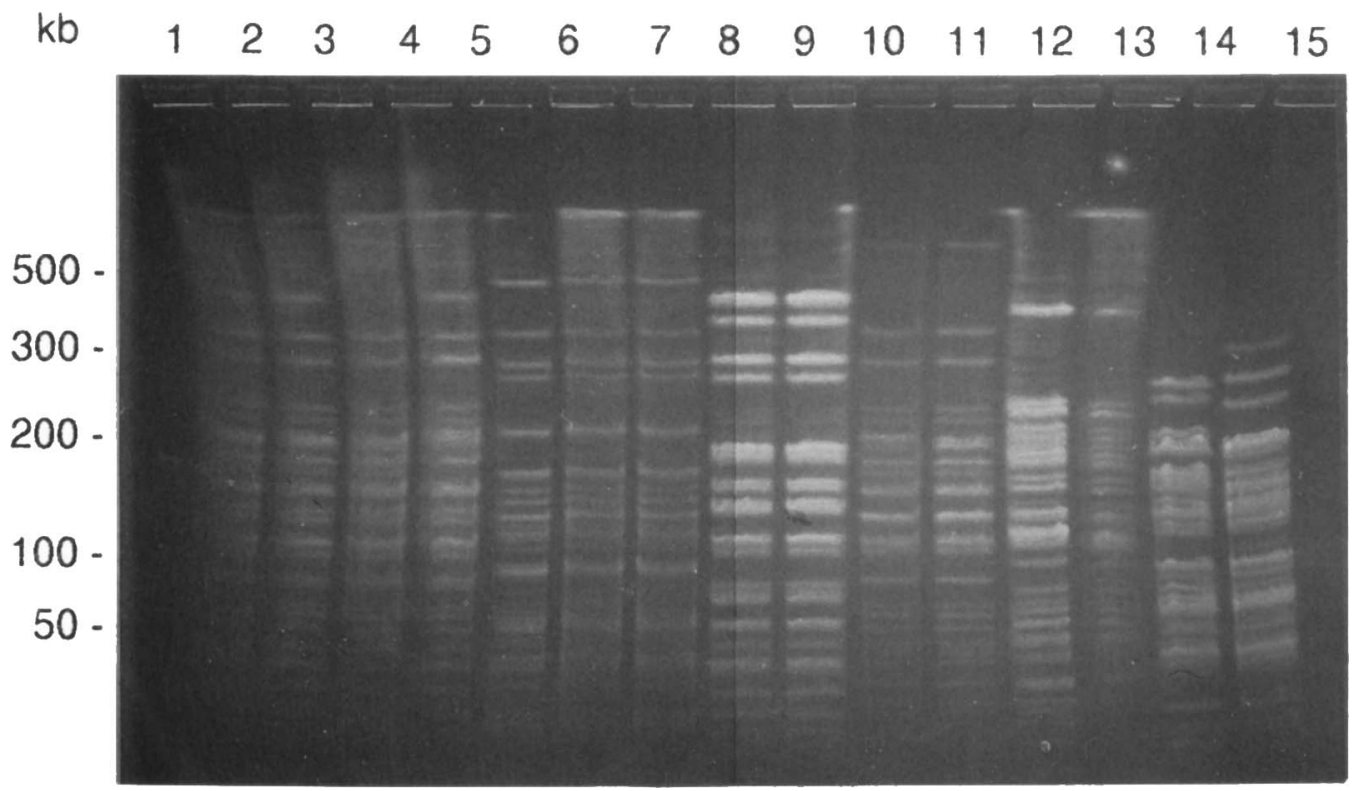

Fig. 3. Macro-restriction analysis of $A$. baumannii chromosomal DNA after digestion with ApaI and PFGE. 
characterisation. To date, reliable genotyping results such as macro-restriction analysis, ribotyping or plasmid analysis become available only after several days and the workload imposed by such techniques rarely justifies their application for routine epidemiological investigations. ${ }^{13}$

RAPD with an M13 single primer has been applied successfully to the typing of $A$. baumannii $i^{15,16}$ and a visual comparison of ethidium bromide-stained agarose gels allowed a differentiation of strains after 2 days. The combination of RAPD with automated laser fluorescence analysis (ALFA) described in the present study is able to generate genotyping results for up to 40 strains within 5-8 h. A high resolution of the amplification pattern is achieved by the use of high-speed denaturing sequencing gels able to discriminate between products that differ by only a single base. ${ }^{17}$ Thus, DNA fingerprints consisting of complex banding patterns of small fragments can be analysed more accurately than by conventional agarose gel electrophoresis. The digitised format of the sequencer data allows consecutive strain alignment and generation of dendrograms without the loss of any information that inadvertently occurs during the scanning of gel images. Moreover, inconsistencies within the gel matrix or variations in its physical properties during a single run that may result in discrepant migration between different lanes can be corrected automatically with the use of internal standards added to all lanes as references, as used in the present study. Therefore, data can be generated and stored as a reference library for comparison with future strains. Cancilla and coworkers have described the use of a DNA sequencer for the typing of bacteria of mainly industrial importance.$^{18}$ However, they were unable to provide rapid results, essential for routine epidemiological screening and early intervention in outbreaks.

\section{References}

1. van Belkum A. DNA fingerprinting of medically important microorganisms by use of PCR. Clin Microbiol Rev 1994; 7: 174-184.

2. Welsh J, McClelland M. Fingerprinting genomes using PCR with arbitrary primers. Nucleic Acids Res 1990; 18: 7213-7218.

3. Caetano-Anollés G, Bassam BJ, Gresshoff PM. DNA amplification fingerprinting using very short arbitrary oligonucleotide primers. Bio/Technology 1991; 9: 553-557.

4. Struelens MJ, Carlier E, Maes N, Serruys E, Quint WGV, van Belkum A. Nosocomial colonization and infection with multiresistant Acinetobacter baumannii: outbreak delineation using DNA macrorestriction analysis and PCRfingerprinting. J Hosp Infect 1993; 25: 15-32.

5. Tenover FC, Arbeit R, Archer $G$ et al. Comparison of traditional and molecular methods of typing isolates of Staphylococcus aureus. J Clin Microbiol 1994; 32: 407-415.

6. Grundmann H, Schneider C, Hartung D, Daschner FD, Pitt TL. Discriminatory power of three DNA-based typing techniques for Pseudomonas aeruginosa. J Clin Microbiol $1995 ; 33$ : $528-534$.

7. Arbeit RD, Maslow JN, Mulligan ME. Polymerase chain reaction-mediated genotyping in microbial epidemiology. Reply. Clin Infect Dis 1994; 18: 1018-1019.

8. Meunier JR, Grimont PAD. Factors affecting reproducibility of random amplified polymorphic DNA fingerprinting. Res Microbiol 1993: 144: 373-379.
The present study showed that RAPD fluorescence profiles were reproducible. Analysis of all isolates in duplicate yielded intra-strain similarity values of $>91 \%$ which gives an estimate of the variance inherent to the technique when amplification products of up to $1000 \mathrm{bp}$ are included in the analysis. This limitation can be explained by inconsistent amplification of larger amplicons, as described by Vila et al. ${ }^{16}$ but reproducibility could be increased to $>95 \%$ if similarity analyses were confined to products of $<700 \mathrm{bp}$. When similarity levels corresponding to those of duplicates were used to indicate genotypic homology, isolates from different patients could be assigned to distinct genotype clusters by RAPDALFA. Moreover, the difference between clusters $(<72 \%$ similarity) allowed an unambiguous discrimination between unrelated strains. Given that the genetic diversity of natural populations of microorganisms represent a continuum, we are currently unable to define similarity levels that could unequivocally discriminate between more closely related strains. Nevertheless, strain delineation obtained by RAPD-ALFA was corroborated by the results of the macro-restriction analysis for all 15 isolates tested.

In conclusion, genetically homologous strains and nosocomial transmissions of $A$. baumannii were identified with a novel combination of automated laser fluorescence analysis, RAPD-fingerprinting and advanced data analysis. In the absence of an outbreak, transmission between no more than two or three patients would usually have gone undetected. In our opinion, this approach may satisfy the criteria of an ideal typing technique, ${ }^{19}$ once the cost-effectiveness of the technique, with its capacity for routine and ongoing molecular typing of isolates of a broad range of bacterial species, e.g., from intensive care units, has been established.

9. Bouvet PJM, Grimont PAD. Taxonomy of the genus Acinetobacter with the recognition of Acinetobacter baumannii sp. nov., Acinetobacter haemolyticus sp. nov., Acinetobacter johnsonii sp. nov., and Acinetobacter junii sp. nov. and emended descriptions of Acinetobacter calcoaceticus and Acinetobacter lwoffii. Int J Syst Bacteriol 1986; 36: $228-240$.

10. Barry T, Powell R, Gannon F. A general method to generate DNA probes for microorganisms. Bio/Technology 1990;8: 233-236.

11. Ansorge W, Sproat BS, Stegemann J, Schwager C. A nonradioactive automated method for DNA sequence termination. J Biochem Biophys Med 1986; 13: 315-323.

12. Beck-Sague CM, Jarwis WR, Brook JH et al. Epidemic bacteremia due to Acinetobacter baumannii in five intensive care units. Am J Epidemiol 1990; 132: 723-733.

13. Seifert H, Boullion B, Schulze A, Pulverer G. Plasmid DNA profiles of Acinetobacter baumannii: clinical application in a complex endemic setting. Infect Control Hosp Epidemiol 1994 ; 15 : $520-528$.

14. Stone JW, Das BC. Investigation of an outbreak of infection with Acinetobacter calcoaceticus in a special care baby unit. J Hosp Infect 1986; 7 : 42-48.

15. Gräser Y, Klare I, Halle E et al. Epidemiological study of an Acinetobacter baumannii outbreak by using polymerase chain reaction fingerprinting. J Clin Microbiol 1993; 31: 2417-2420.

16. Vila J, Marcos A, Llovet T, Coll P, Jimenez de Anta T. A comparative study of ribotyping and arbitrarily primed 
polymerase chain reaction for investigation of hospital outbreaks of Acinetobacter baumannii infection. $J$ Med Microbiol 1994; 41: 244-249.

17. Voss H, Stegemann J, Schwager C et al. High-speed automated DNA fragment analysis for genome mapping by restriction fingerprinting. Methods Mol Cell Biol 1992; 3: 77-82.

18. Cancilla MR, Powell JB, Hillier AJ, Davidson BE. Rapid genomic fingerprinting for Lactococcus lactis strains by arbitrarily primed polymerase chain reaction with ${ }^{32} \mathrm{P}$ and fluorescent labels. Appl Environ Microbiol 1992; 58: 1772-1775.

19. Maslow JN, Mulligan ME, Arbeit RD. Molecular epidemiology: application of contemporary techniques to typing of microorganisms. Clin Infect Dis 1993; 17: 153-164. 